\title{
Front Matter: Volume 8683
}

, "Front Matter: Volume 8683," Proc. SPIE 8683, Optical Microlithography XXVI, 868301 (23 April 2013); doi: 10.1117/12.2028885

SPIE. Event: SPIE Advanced Lithography, 2013, San Jose, California, United States 


\section{PROCEEDINGS OF SPIE}

\section{Optical Microlithography XXVI}

Will Conley

Editor

\section{6-28 February 2013}

San Jose, California, United States

Sponsored

SPIE

Cosponsored by

Cymer, Inc. (United States)

Published by

SPIE 
The papers included in this volume were part of the technical conference cited on the cover and title page. Papers were selected and subject to review by the editors and conference program committee. Some conference presentations may not be available for publication. The papers published in these proceedings reflect the work and thoughts of the authors and are published herein as submitted. The publisher is not responsible for the validity of the information or for any outcomes resulting from reliance thereon.

Please use the following format to cite material from this book: Author(s), "Title of Paper," in Optical Microlithography XXVI, edited by Will Conley, Proceedings of SPIE Vol. 8683 (SPIE, Bellingham, WA, 2013) Article CID Number.

ISSN: 0277-786X

ISBN: 9780819494658

Published by

SPIE

P.O. Box 10, Bellingham, Washington 98227-0010 USA

Telephone +1 3606763290 (Pacific Time) · Fax +1 3606471445

SPIE.org

Copyright $@ 2013$, Society of Photo-Optical Instrumentation Engineers.

Copying of material in this book for internal or personal use, or for the internal or personal use of specific clients, beyond the fair use provisions granted by the U.S. Copyright Law is authorized by SPIE subject to payment of copying fees. The Transactional Reporting Service base fee for this volume is $\$ 18.00$ per article (or portion thereof), which should be paid directly to the Copyright Clearance Center (CCC), 222 Rosewood Drive, Danvers, MA 01923. Payment may also be made electronically through CCC Online at copyright.com. Other copying for republication, resale, advertising or promotion, or any form of systematic or multiple reproduction of any material in this book is prohibited except with permission in writing from the publisher. The CCC fee code is $0277-786 \mathrm{X} / 13 / \$ 18.00$.

Printed in the United States of America.

Publication of record for individual papers is online in the SPIE Digital Library.

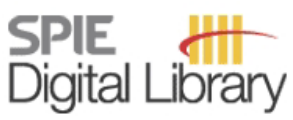

SPIEDigitalLibrary.org

Paper Numbering: Proceedings of SPIE follow an e-First publication model, with papers published first online and then in print and on CD-ROM. Papers are published as they are submitted and meet publication criteria. A unique, consistent, permanent citation identifier (CID) number is assigned to each article at the time of the first publication. Utilization of CIDs allows articles to be fully citable as soon as they are published online, and connects the same identifier to all online, print, and electronic versions of the publication. SPIE uses a six-digit CID article numbering system in which:

- The first four digits correspond to the SPIE volume number.

- The last two digits indicate publication order within the volume using a Base 36 numbering

system employing both numerals and letters. These two-number sets start with 00, 01, 02, 03, 04,

05, 06, 07, 08, 09, 0A, OB ... 0Z, followed by 10-1Z, 20-2Z, etc.

The CID Number appears on each page of the manuscript. The complete citation is used on the first page, and an abbreviated version on subsequent pages. Numbers in the index correspond to the last two digits of the six-digit CID Number. 


\section{Contents}

868304 Computational aspects of optical lithography extension by directed self-assembly [8683-3] K. Lai, IBM Corp. (United States); C. Liu, IBM Albany Nano Technology Ctr. (United States); J. Pitera, IBM Almaden Research Ctr. (United States); D. J. Dechene, IBM Corp. (United States); A. Schepis, J. Abdallah, IBM Albany Nano Technology Ctr. (United States); H. Tsai, M. Guillorn, IBM Yorktown Research Ctr. (United States); J. Cheng, G. Doerk, M. Tjio, C. Rettner, IBM Almaden Research Ctr. (United States); O. Odesanya, M. Ozlem, N. Lafferty, IBM Corp. (United States)

868305 Sub-12nm optical lithography with $4 x$ pitch division and SMO-lite [8683-4] M. C. Smayling, Tela Innovations, Inc. (United States); K. Tsujita, Canon, Inc. (Japan); H. Yaegashi, Tokyo Electron, Ltd. (Japan); V. Axelrad, Sequoia Design Systems, Inc. (United States); T. Arai, Canon, Inc. (Japan); K. Oyama, A. Hara, Tokyo Electron, Ltd. (Japan)

868306 Impact of process decisions and alignment strategy on overlay for the 14nm node [8683-5] D. Laidler, K. D'havé, P. Leray, J. Hermans, J. Boemmels, S. Cheng, IMEC (Belgium); H. Dai, Y. Chen, B. Mebarki, C. Ngai, Applied Materials, Inc. (United States)

868307 The impact of 14-nm photomask uncertainties on computational lithography solutions [8683-6]

J. Sturtevant, E. Tejnil, T. Lin, S. Schultze, Mentor Graphics Corp. (United States); P. Buck, F. Kalk, K. Nakagawa, Toppan Photomasks, Inc. (United States); G. Ning, P. Ackmann, GLOBALFOUNDRIES Inc. (United States); F. Gans, C. Buergel, Advanced Mask Technology Ctr. (Germany)

868308 An investigation into scalability and compliance for triple patterning with stitches for metal 1 at the $14 \mathrm{~nm}$ node [8683-7]

C. Cork, Synopsys SARL (France); A. Miloslavsky, P. Friedberg, G. Luk-Pat, Synopsys, Inc. (United States)

SOURCE AND MASK OPTIMIZATION (SMO) I

868309

Robust SMO methodology for exposure tool and mask variations in high volume production [8683-8]

T. Hashimoto, Y. Kai, K. Masukawa, S. Nojima, T. Kotani, Toshiba Corp. (Japan)

8683 0A Imaging application tools for extremely low-k1 ArF immersion lithography [8683-9]

S. Mori, H. Aoyama, T. Ogata, R. Matsui, T. Matsuyama, Nikon Corp. (Japan) 
8683 OB Study of recent CFD-based scheme for analyzing 3D mask effects [8683-10]

M. Takahashi, K. Kodera, M. Motokubota, Toshiba Corp. (Japan); Y. Kawabata, Toshiba Information Systems Corp. (Japan); S. Maeda, S. Nojima, S. Tanaka, S. Mimotogi, Toshiba Corp. (Japan)

$86830 \mathrm{C}$ The effect of mask and source complexity on source-mask optimization [8683-11] S.-H. Yang, N. Jia, S. B. Shim, D. Vengertsev, Y. Kim, J. Choi, H.-K. Kang, Samsung Electronics Co., Ltd. (Korea, Republic of)

8683 OD Illumination pupilgram control using an intelligent illuminator [8683-12]

N. Hirayanagi, Y. Mizuno, M. Mori, N. Kita, R. Matsui, T. Matsuyama, Nikon Corp. (Japan)

RET

$8683 \mathrm{OE}$ Inverse lithography technique for advanced CMOS nodes [8683-14]

A. Villaret, STMicroelectronics (France); A. Tritchkov, Mentor Graphics Corp. (United States); J. Entradas, Mentor Graphics Ltd. (France); E. Yesilada, STMicroelectronics (France)

$8683 \mathrm{OF}$ Mask compensation for process flare in 193nm very low k1 lithography [8683-15] J. Lee, T. Lee, S. Oh, C. Kang, J. Kim, J. Choi, C. Park, H. Yang, D. Yim, SK Hynix, Inc. (Korea, Republic of); M. Do, Synopsys, Inc. (Korea, Republic of); I. Su, Synopsys, Inc. (Taiwan); H. Song, Synopsys, Inc. (United States); J.-H. Choi, Synopsys, Inc. (Korea, Republic of); Y. Fan, A. C. Wang, Synopsys, Inc. (United States); S.-W. Lee, Synopsys, Inc. (Korea, Republic of); R. Boone, K. Lucas, Synopsys, Inc. (United States)

8683 OG Pupil wavefront manipulation to compensate for mask topography effects in optical nanolithography [8683-16]

M. Kempsell Sears, B. W. Smith, Rochester Institute of Technology (United States)

$8683 \mathrm{OH}$ Effective model-based SRAF placement for full chip 2D layouts [8683-17]

S. Jayaram, P. LaCour, J. Word, A. Tritchkov, Mentor Graphics Corp. (United States)

8683 ol Wafer topography modeling for ionic implantation mask correction dedicated to $2 \mathrm{x} \mathrm{nm}$ FDSOI technologies [8683-18]

J.-C. Michel, J.-C. Le Denmat, E. Sungauer, F. Robert, E. Yesilada, STMicroelectronics

(France); A.-M. Armeanu, J. Entradas, Mentor Graphics Ltd. (France); J. L. Sturtevant, T. Do, Y. Granik, Mentor Graphics Corp. (United States)

SOURCE AND MASK OPTIMIZATION (SMO) II

$86830 \mathrm{~J}$ Enabling reverse tone imaging for via levels using attenuated phase shift mask and source optimization [8683-19]

B. Hamieh, STMicroelectronics (United States); H. C. Choi, Samsung Electronics Co., Ltd. (United States); B. Erenturk, GLOBALFOUNDRIES Inc. (United States); W. Guo, IBM Corp. (United States); A. Hamouda, H. Liu, GLOBALFOUNDRIES Inc. (United States); G. McIntyre, J. Meiring, IBM Corp. (United States); D. Moreau, STMicroelectronics (United States);

A. Thomas, A. Wei, IBM Corp. (United States) 
8683 OK Introducing a novel flow to estimate challenges encountered while transitioning from RET development to manufacturable solution [8683-20]

J. Cheng, GLOBALFOUNDRIES Inc. (Singapore); R. Chia, Mentor Graphics Corp. (United States); Y. Gong, GLOBALFOUNDRIES Inc. (Singapore); O. ElSewefy, Mentor Graphics Corp. (United States); G. S. Chua, Y. M. Foong, GLOBALFOUNDRIES Inc. (Singapore); A. Dave, A. Chua, Mentor Graphics Corp. (United States); D. Q. Zhang, GLOBALFOUNDRIES Inc. (Singapore); V. Liubich, P. Lacour, A. Tritchkov, Mentor Graphics Corp. (United States)

$8683 \mathrm{OL}$ Manufacturability of computation lithography mask: current limit and requirements for sub20nm node [8683-21]

J. Choi, I.-Y. Kang, J. S. Park, I. K. Shin, C.-U. Jeon, Samsung Electronics Co., Ltd. (Korea, Republic of)

$8683 \mathrm{OM}$ The impact of realistic source shape and flexibility on source mask optimization [8683-22] H. Aoyama, Y. Mizuno, N. Hirayanagi, N. Kita, R. Matsui, Nikon Corp. (Japan); H. Izumi, K. Tajima, Nihon Synopsys G. K. (Japan); J. Siebert, W. Demmerle, Synopsys GmbH (Germany); T. Matsuyama, Nikon Corp. (Japan)

$8683 \mathrm{ON}$ Source and mask optimization to mitigate hotspots in etch process [8683-23] Y. Kono, Y. Kai, K. Masukawa, S. Tamaoki, T. Hashimoto, T. Kimura, R. Aburada, T. Kotani, Toshiba Corp. (Japan)

868300 Global source optimization for MEEF and OPE [8683-24]

R. Matsui, T. Noda, H. Aoyama, N. Kita, T. Matsuyama, Nikon Corp. (Japan); D. Flagello, Nikon Research Corp. of America (United States)

\section{PROCESS TECHNOLOGY I}

$8683 \mathrm{OP}$ Integrated scatterometry for tight overlay and CD control to enable $20-\mathbf{n m}$ node wafer manufacturing. [8683-25]

J. Benschop, A. Engelen, H. Cramer, M. Kubis, P. Hinnen, H. van der Laan,

K. Bhattacharyya, J. Mulkens, ASML Netherlands B.V. (Netherlands)

$86830 Q$ Mix-and-match overlay performance of the NSR-S622D immersion scanner [8683-26] K. Makino, T. Kikuchi, S. Sasamoto, P. Hongki, A. Mori, N. Takahashi, S. Wakamoto, Nikon Corp. (Japan)

$8683 \mathrm{OR}$ Lithographic challenges and their solutions for critical layers in sub-14nm node logic devices [8683-92]

T.-B. Chiou, ASML (Taiwan); M. Dusa, ASML (Belgium); A. C. Chen, ASML (Taiwan);

D. Pietromonaco, ARM Ltd. (United States)

8683 OS Lithography imaging control by enhanced monitoring of light source performance [8683-28]

P. Alagna, Cymer, Inc. (United States) and Cymer, Inc. (Belgium); O. Zurita, I. Lalovic, N. Seong, G. Rechsteiner, J. Thornes, Cymer, Inc. (Belgium); K. D'havé, L. Van Look, J. Bekaert, IMEC (Belgium) 
8683 OU Solutions with precise prediction for thermal aberration error in low-k1 immersion lithography [8683-29]

K. Fukuhara, A. Mimotogi, T. Kono, Toshiba Corp. (Japan); H. Aoyama, T. Ogata, N. Kita, T. Matsuyama, Nikon Corp. (Japan)

8683 OV Compact OPC model optimization using emulated data [8683-30]

A. Isoyan, Synopsys, Inc. (United States); T. Mülders, Synopsys GmbH (Germany);

C. Westwood, L. S. Melvin III, Synopsys, Inc. (United States)

8683 oW A study on the automation of scanner matching [8683-31]

Y. He, Micron Technology, Inc. (United States); A. Serebryakov, ASML US, Inc. (United

States); S. Light, Micron Technology, Inc. (United States); V. Jain, ASML US, Inc. (United

States); E. Byers, Micron Technology, Inc. (United States); R. Goossens, Z.-Y. Niu, P. Engblom, S. Larson, ASML US, Inc. (United States); B. Geh, Carl Zeiss SMT Inc. (United States);

C. Hickman, H. Kang, Micron Technology, Inc. (United States)

8683 OX Adjustment of image decomposition mode and reflection criterion focusing on critical dimension uniformity and exposure dose effectiveness under diffraction effects in optical microlithography using a digital micromirror device [8683-32]

M. Seo, H. Kim, Tongmyong Univ. (Korea, Republic of)

8683 OY Simulation of spacer-based SADP (Self-Aligned Double-Patterning) for $15 \mathrm{~nm}$ half pitch [8683-33]

S. Robertson, KLA-Tencor Texas (United States); P. Wong, J. Versluijs, V. Wiaux, IMEC

(Belgium)

\section{PROCESS TECHNOLOGY II}

$86830 Z$ A comparative study of self-aligned quadruple and sextuple patterning techniques for sub-15nm IC scaling [8683-34]

Y. Chen, W. Kang, P. Zhang, Peking Univ. (China)

868310 Grayscale lithography: 3D structuring and thickness control [8683-35]

M. Heller, D. Kaiser, M. Stegemann, G. Holfeld, N. Morgana, J. Schneider, D. Sarlette, Infineon Technologies Dresden GmbH (Germany)

868311 Sidewall profile inclination modulation mask (SPIMM): modification of an attenuated phase-shift mask for single-exposure double and multiple patterning [8683-36]

F. T. Chen, W.-S. Chen, M.-J. Tsai, T.-K. Ku, Industrial Technology Research Institute (Taiwan)

868312 Avoiding wafer-print artifacts in spacer is dielectric (SID) patterning [8683-37]

G. Luk-Pat, B. Painter, A. Miloslavsky, Synopsys, Inc. (United States); P. De Bisschop, IMEC

(Belgium); A. Beacham, Synopsys, Inc. (Canada); K. Lucas, Synopsys, Inc. (United States)

868313 Best focus shift mitigation for extending the depth of focus [8683-38]

A. Szucs, J. Planchot, V. Farys, E. Yesilada, C. Alleaume, STMicroelectronics (France);

L. Depre, R. Dover, ASML Brion (United States); C. Gourgon, M. Besacier, LTM, CNRS, CEA

(France); A. Nachtwein, P. Rusu, ASML Netherland B.V. (Netherlands) 
868314 Wafer sub-layer impact in OPC/ORC models for $2 \times \mathbf{n m}$ node implant layers [8683-39] J.-C. Le-Denmat, C. Martinelli, E. Sungaver, J.-C. Michel, E. Yesilada, F. Robert,

STMicroelectronics (France)

OPTICAL AND DFM I: JOINT SESSION WITH CONFERENCES 8683 AND 8684

868315 Interference harmonics and rigorous EM spectrum analysis method for low-k1 CD Bossung tilt correction [8683-40]

S.-Y. Chou, H.-T. Ng, Y.-Y. Chen, C.-F. Lee, R.-G. Liu, T.-S. Gau, Taiwan Semiconductor Manufacturing Co. Ltd. (Taiwan)

868316 Model based stitching and inter-mask bridge prevention for double patterning lithography [8683-41]

G. Landié, J.-N. Pena, STMicroelectronics (France); S. Postnikov, Mentor Graphics Corp.

(France); J. Word, S. Shang, Mentor Graphics Corp. (United States); F. Chaovi, Mentor

Graphics Corp. (France); E. Yesilada, C. Martinelli, STMicroelectronics (France)

868317 Application of artificial neural networks to compact mask models in optical lithography simulation (Best Student Paper Award) [8683-42]

V. Agudelo, Friedrich-Alexander-Univ. Erlangen-Nürnberg (Germany) and FraunhoferInstitut für Integrierte Systeme und Bavelementetechnologie (Germany); T. Fühner, Fraunhofer-Institut für Integrierte Systeme und Bavelementetechnologie (Germany); A. Erdmann, Friedrich-Alexander-Univ. Erlangen-Nürnberg (Germany) and FraunhoferInstitut für Integrierte Systeme und Bavelementetechnologie (Germany); P. Evanschitzky, Fraunhofer-Institut für Integrierte Systeme und Bavelementetechnologie (Germany)

OPTICAL AND DFM II: JOINT SESSION WITH CONFERENCES 8683 AND 8684

868318 3D resist profile modeling for OPC applications [8683-43]

Y. Fan, Synopsys, Inc. (United States); K. K. Koh, Q. Yang, GLOBALFOUNDRIES Inc.

(Singapore); W. Hoppe, B. Kuechler, Synopsys GmbH (Germany); P. Perampalam, M. Miyagi, L. Zavyalova, Synopsys, Inc. (United States); T. Schmöller, Synopsys GmbH (Germany)

868319 On the accuracy of different Fourier transforms of VLSI designs [8683-44]

R. Nasser, P. Hurley, IBM Zürich Research Lab (Switzerland)

SIMULATION

8683 1 A Benchmarking study of 3D mask modeling for 2X and 1X nodes [8683-45]

C. A. Wang, C.-C. Liang, H. Liu, C. Kallingal, GLOBALFOUNDRIES Inc. (United States);

D. Dunn, J. Oberschmidt, IBM Corp. (United States); J. Tirapu Azpiroz, IBM Corp. (Brazil)

8683 IB OPC resist model separability validation after SMO source change [8683-46]

W. Gillijns, J. Van de Kerkhove, D. Trivkovic, P. De Bisschop, IMEC (Belgium); D. Rio, S. Hsu,

M. Feng, Q. Zhang, H. Liu, ASML Brion (United States) 
8683 IC Topographic mask modeling with reduced basis finite element method [8683-47] J. K. Tyminski, Nikon Research Corp. of America (United States); J. Pomplun, L. Zschiedrich, JCMwave GmbH (Germany); D. Flagello, Nikon Research Corp. of America (United States): T. Matsuyama, Nikon Corp. (Japan)

8683 ID Accurate 3DEMF mask model for full-chip simulation [8683-48] M. C. Lam, K. Adam, D. Fryer, C. Zuniga, H. Wei, M. Oliver, C. H. Clifford, Mentor Graphics Corp. (United States)

$8683 \mathrm{IE} \quad$ Role of 3D photo-resist simulation for advanced technology nodes [8683-49] A. Narayana Samy, R. Seltmann, F. Kahlenberg, J. Schramm, GLOBALFOUNDRIES Inc. (Germany); B. Küchler, U. Klostermann, Synopsys GmbH (Germany)

TOOLING

8683 IF A study of vertical lithography for high-density 3D structures [8683-50] M. Mizutani, S.-I. Hirai, I. Koizumi, K.-I. Mori, S. Miura, Canon Inc. (Japan)

8683 1G Power up: 120 Watt injection-locked ArF excimer laser required for both multi-patterning and $\mathbf{4 5 0} \mathbf{m m}$ wafer lithography [8683-51]

T. Asayama, Y. Sasaki, T. Nagashima, A. Kurosu, H. Tsushima, T. Kumazaki, K. Kakizaki,

T. Matsunaga, H. Mizoguchi, Gigaphoton Inc. (Japan)

8683 1H High power 120W ArF immersion XLR laser system for high dose applications [8683-52]

R. Rokitski, R. Rafac, J. Melchior, R. Dubi, J. Thornes, T. Cacouris, M. Haviland, D. Brown, Cymer, Inc. (United States)

868311 Comprehensive thermal aberration and distortion control of lithographic lenses for accurate overlay [8683-53]

Y. Fujishima, S. Ishiyama, S. Isago, A. Fukui, H. Yamamoto, T. Hirayama, T. Matsuyama, Y. Ohmura, Nikon Corp. (Japan)

8683 1 J High order field-to-field corrections for imaging and overlay to achieve sub 20-nm lithography requirements [8683-54] J. Mulkens, M. Kubis, P. Hinnen, R. de Graaf, H. van der Laan, A. Padiy, B. Menchtchikov, ASML Netherlands B.V. (Netherlands)

8683 1K High-productivity immersion scanner enabling 1xnm hp manufacturing [8683-55] Y. Shirata, Y. Shibazaki, J. Kosugi, T. Kikuchi, Y. Ohmura, Nikon Corp. (Japan)

$8683 \mathrm{lL} \quad$ Extending immersion lithography down to $1 \mathrm{x} \mathrm{nm}$ production nodes [8683-91] W. de Boeij, R. Pieternella, I. Bouchoms, M. Leenders, M. Hoofman, R. de Graaf, H. Kok, P. Broman, J. Smits, J.-J. Kuit, M. McLaren, ASML Netherlands B.V. (Netherlands) 
$86831 \mathrm{M}$ Compatibility of optimized source over design changes in the foundry environment [8683-56]

J. Pei, Semiconductor Manufacturing International Corp. (China); F. Shao, O. ElSewefy, C. Zhu, Mentor Graphics Corp. (United States); V. XU, Semiconductor Manufacturing International Corp. (China); Y. Zhu, L. Zhang, Mentor Graphics Corp. (United States); X. Shi, Q. Liu, Semiconductor Manufacturing International Corp. (China); A. Dave, Mentor Graphics Corp. (United States)

8683 IN 3D resist profile full chip verification and hot spot disposition [8683-57]

Q. Yang, S. F. Quek, Y. M. Foong, GLOBALFOUNDRIES Inc. (Singapore); J. Hassmann, GLOBALFOUNDRIES Inc. (Germany); D. Zhang, GLOBALFOUNDRIES Inc. (Singapore); A. Leschok, GLOBALFOUNDRIES Inc. (Germany); T. Yun, GLOBALFOUNDRIES Inc. (Singapore); M. Feng, S. Baron, J. H. Qiu, T. Pandey, B. Yan, R. Dover, ASML Brion Technologies (United States)

8683 IP 3D lithography for implant applications [8683-59]

J. Schneider, H. Feick, D. Kaiser, M. Heller, D. Sarlette, Infineon Technologies Dresden GmbH (Germany)

$86831 Q$ Lens heating impact analysis and controls for critical device layers by computational method [8683-60]

D. H. Beak, J. P. Choi, T. Park, Y. S. Nam, Y. S. Kang, C. H. Park, Samsung Electronics Co., Ltd. (Korea, Republic of); K.-Y. Park, C.-H. Ryu, W. Huang, K.-H. Baik, ASML Korea Co., Ltd. (Korea, Republic of)

8683 IR Effects of focus difference of nested and isolated features for scanner proximity matching [8683-61]

G. Ning, GLOBALFOUNDRIES Inc. (United States); and GLOBALFOUNDARIES Inc. (Germany); P. Ackmann, H. Koh, GLOBALFOUNDRIES Inc. (United States); F. Richter, M. Ruhm, J. Busch, GLOBALFOUNDRIES Inc. (Germany); N. Chen, GLOBALFOUNDRIES Inc. (United States); K. Kurth, A. Leschok, GLOBALFOUNDRIES Inc. (Germany); C. T. Lim, GLOBALFOUNDRIES Inc. (United States)

8683 IT Source mask optimization using real-coded genetic algorithms [8683-63] C. Yang, X. Wang, S. Li, Shanghai Institute of Optics and Fine Mechanics (China) and Graduate School of the Chinese Academy of Sciences (China); A. Erdmann, FraunhoferInstitut für Integrierte Systeme und Bauelementetechnologie (Germany)

8683 IV Mask 3D effects on contact layouts of 1Xnm NAND flash devices [8683-65] J. Jang, H. Jeong, H. Yune, S. Oh, H. Yang, D. Yim, SK Hynix, Inc. (Korea, Republic of)

8683 IW High speed and flexible PEB 3D diffusion simulation based on Sylvester equation [8683-66] P.-C. Lin, C. C-P. Chen, National Taiwan Univ. (Taiwan)

8683 IY Line edge roughness (LER) mitigation studies specific to interference-like lithography [8683-68]

B. Baylav, A. Estroff, Rochester Institute of Technology (United States); P. Xie, Applied Materials, Inc. (United States); B. W. Smith, Rochester Institute of Technology (United States) 
$86831 \mathrm{~T}$ The studies of SMO process on cont layer of 20nm node [8683-69]

W. C. Lo, Y. F. Cheng, M. J. Chen, United Microelectronics Corp. (Taiwan)

868322 SMO and NTD for robust single exposure solution on contact patterning for 40nm node flash memory devices [8683-72]

C.-C. Yu, C. C. Yang, E. Yang, T. H. Yang, K. C. Chen, C.-Y. LU, Macronix International Co., Ltd. (Taiwan)

868323 Multiple-step process window aware OPC for hyper-NA lithography [8683-73]

C. T. Hsuan, C. M. Hu, F. Lo, E. Yang, T. H. Yang, K. C. Chen, C.-Y. Lu, Macronix International Co., Ltd. (Taiwan)

868324 Studies of a suitable mask error enhancement factor for 2D patterns [8683-74]

C. I. Wei, Y. F. Cheng, M. J. Chen, United Microelectronics Corp. (Taiwan)

868325 Pixel-based inverse lithography using a mask filtering technique [8683-75]

W. Lv, Q. Xia, S. Liu, Huazhong Univ. of Science and Technology (China)

868326 Imaging quality full chip verification for yield improvement [8683-76]

Q. Yang, C. S. Zhou, S. F. Quek, M. Lu, Y. M. Foong, GLOBALFOUNDRIES Inc. (Singapore);

J. H. Qiu, T. Pandey, R. Dover, ASML Brion Technologies (United States)

$868327 \quad$ Hybrid OPC technique using model based and rule-based flows [8683-77]

M. Harb, H. Abdelghany, Mentor Graphics Egypt (Egypt)

868329 Model of freeform illumination mode and polarization mode for 193nm immersion lithographic machine [8683-80]

Y. Zhang, A. Zeng, Y. Wang, M. Chen, H. Huang, Shanghai Institute of Optics and Fine

Mechanics (China)

8683 2A Analytical equation predicting the forbidden pattern pitch for phase-shiftimg mask [8683-81]

J. Tamaki, Tokyo Polytechnic Univ. (Japan) and Orc Manufacturing Co., Ltd. (Japan);

M. Shibuya, N. Suezou, Tokyo Polytechnic Univ. (Japan)

8683 2C Optomechanical characterization of large wafer stepper-optics with respect to centering errors, lens distances, and center thicknesses [8683-83]

D. Stickler, P. Langehanenberg, B. Lüerß, J. Heinisch, TRIOPTICS GmbH (Germany)

8683 2D A customized Exicor system for measuring residual birefringence in lithographic lenses [8683-84]

A. Breninger, B. Wang, Hinds Instruments, Inc. (United States)

$86832 \mathrm{E} \quad$ Flare management for 40-nm logic devices [8683-85]

Y. Tanaka, T. Tamura, M. Fujimoto, K. Tsubata, N. Onoda, K. Fujii, Renesas Electronics Corp. (Japan)

$86832 \mathrm{~F}$ Wavefront testing of pinhole based on point diffraction interferometer [8683-86]

X. Jin, T. Xing, J. Xu, W. Lin, Z. Liao, Institute of Optics and Electronics (China) 
$86832 G$ Design and simulation of illuminator with micro scanning slit array for NA 0.75 lithography system [8683-87]

L. Zhu, Shanghai Institute of Optics and Fine Mechanics (China); A. Zeng, Shanghai Institute of Optics and Fine Mechanics (China) and Univ. of the Chinese Academy of Sciences (China); S. Zhang, Shanghai Institute of Optics and Fine Mechanics (China); R. Fang, H. Huang, Shanghai Institute of Optics and Fine Mechanics (China) and Univ. of the Chinese Academy of Sciences (China)

$86832 \mathrm{H} \quad$ Zero expansion glass ceramic ZERODUR ${ }^{8}$ roadmap for advanced lithography [8683-88] T. Westerhoff, R. Jedamzik, P. Hartmann, SCHOTT AG (Germany)

8683 2I Mask side wall clamping [8683-89]

G. J. P. Naaijkens, P. C. J. N. Rosielle, M. Steinbuch, Technische Univ. Eindhoven (Netherlands)

Author Index 


\section{Conference Committee}

Symposium Chair

Harry J. Levinson, GLOBALFOUNDRIES, Inc. (United States)

Symposium Cochair

Mircea V. Dusa, ASML US, Inc. (United States)

Conference Chair

Will Conley, Cymer, Inc. (United States)

Conference CoChair

Kafai Lai, IBM Corporation (United States)

Conference Program Committee

Pary Baluswamy, Micron Technology, Inc. (United States)

Peter D. Brooker, Synopsys, Inc. (United States)

Peter D. Buck, Toppan Photomasks, Inc. (United States)

Andreas Erdmann, Fraunhofer-Institut für Integrierte System und

Bavelementetechnologie (Germany)

Nigel R. Farrar, Cymer, Inc. (United States)

Jo Finders, ASML Netherlands B.V. (Netherlands)

Carlos Fonseca, Tokyo Electron America, Inc. (United States)

Tsai-Sheng Gau, Taiwan Semiconductor Manufacturing Company,

Ltd. (Taiwan)

Bernd Geh, Carl Zeiss SMT Inc. (United States)

Yuri Granik, Mentor Graphics Corporation (United States)

Sachiko Kobayashi, Toshiba Corporation (Japan)

Jongwook Kye, GLOBALFOUNDRIES Inc. (United States)

Sukjoo Lee, Samsung Electronics Company, Ltd. (Korea, Republic of)

Wilhelm Maurer, Infineon Technologies AG (Germany)

Soichi Owa, Nikon Corp. (Japan)

Xuelong Shi, Semiconductor Manufacturing International Corporation (China)

Sam Sivakumar, Intel Corporation (United States)

Bruce W. Smith, Rochester Institute of Technology (United States)

Kazuhiro Takahashi, Canon Inc. (Japan)

Geert Vandenberghe, IMEC (Belgium) 
Session Chairs

1 Keynote Session

Will Conley, Cymer, Inc. (United States)

Kafai Lai, IBM Corporation (United States)

$2 \quad 14 \mathrm{~nm}$ and Beyond

Jongwook Kye, GLOBALFOUNDRIES Inc. (United States)

Tsai-Sheng Gau, Taiwan Semiconductor Manufacturing Company Ltd. (Taiwan)

3 Source and Mask Optimization (SMO) I

Andreas Erdmann, Fraunhofer-Institut für Integrierte Systeme und Bavelementetechnologie (Germany)

Bruce W. Smith, Rochester Institute of Technology (United States)

4 RET

Carlos Fonseca, Tokyo Electron America, Inc. (United States)

Sachiko Kobayashi, Toshiba Materials Company, Ltd. (Japan)

5 Source and Mask Optimization (SMO) II

Kazuhiro Takahashi, Canon Inc. (Japan)

Kurt Ronsed, IMEC (Belgium)

6 Process Technology I

Pary Baluswamy, Micron Technology, Inc. (United States)

Wilhelm Maurer, Infineon Technologies AG (Germany)

7 Modeling

Bernd Geh, Carl Zeiss SMT Inc. (United States)

Xuelong Shi, Semiconductor Manufacturing International Corporation (China)

8 Process Technology II

Sukjoo Lee, Samsung Electronics Company, Ltd. (Korea, Republic of) Nigel R. Farrar, Cymer, Inc. (United States)

9 Optical and DFM I: Joint Session with Conferences 8683 and 8684

Mark E. Mason, Texas Instruments Inc. (United States)

Will Conley, Cymer, Inc. (United States)

10 Optical and DFM II: Joint Session with Conferences 8683 and 8684

Kafai Lai, IBM Corporation (United States)

John L. Sturtevant, Mentor Graphics Corporation (United States) 
11 Simulation

Peter D. Brooker, Synopsys, Inc. (United States)

Yuri Granik, Mentor Graphics Corporation (United States)

12 Tooling

Soichi Owa, Nikon Corporation (Japan)

Jo Finders, ASML Netherlands B.V. (Netherlands)

Proc. of SPIE Vol. $8683868301-14$

Downloaded From: https://www.spiedigitallibrary.org/conference-proceedings-of-spie on 26 Apr 2023 Terms of Use: https://www.spiedigitallibrary.org/terms-of-use 